\title{
Interface-Assisted Sign Inversion of Magnetoresistance in Spin Valves Based on Novel Lanthanide Quinoline Molecules
}

Amilcar Bedoya-Pinto,Sara G. Miralles,Saül Vélez,Ainhoa Atxabal,Pierluigi Gargiani,Manuel Valvidares,Fèlix Casanova,Eugenio Coronado,Luis E. Hueso

\section{Abstract}

Molecules are proposed to be an efficient medium to host spin-polarized carriers, due to their weak spin relaxation mechanisms. While relatively long spin lifetimes are measured in molecular devices, the most promising route toward device functionalization is to use the chemical versatility of molecules to achieve a deterministic control and manipulation of the electron spin. Here, by combining magnetotransport experiments with element-specific X-ray absorption spectroscopy, this study shows the ability of molecules to modify spin-dependent properties at the interface level via metalmolecule hybridization pathways. In particular, it is described how the formation of hybrid states determines the spin polarization at the relevant spin valve interfaces, allowing the control of macroscopic device parameters such as the sign and magnitude of the magnetoresistance. These results consolidate the application of the spinterface concept in a fully functional device platform.

\section{Introduction}

Molecules, due to their wide-ranging chemical functionalities that can be tailored on demand, are becoming increasingly attractive components for applications in materials science and solid-state physics. Remarkable progress has been made in the fields of molecular-based electronics and optoelectronics, with devices such as organic field-effect transistors $\underline{\mathbf{1}}$ and light-emitting diodes. $\underline{\mathbf{2}}, \underline{\mathbf{3}}$ As for spintronics, a nascent field which aims to use the spin of the electron for information processing, molecules have been proposed to be an efficient medium to host spin-polarized carriers due to their weak spin relaxation mechanisms.4-9 Recently, the research focus in this discipline has been set in using the chemical versatility of molecules to achieve a deterministic control and manipulation of the electron spin. Significant progress has been made by controlling and reading spins at the single-molecule level $\underline{\mathbf{1 0}}-\underline{\mathbf{1 2}}$ as well as in large-area spin-valve devices. $\underline{\mathbf{1 3}}$ In the latter case of macroscopic spin valves, there are two main relevant aspects. First, the ability to customize the energy-level alignment by the proper choice of molecules and metal electrodes is a crucial factor to achieve an efficient (spin-polarized) charge injection.14- 
$\underline{18}$ Second, spin-polarized hybrid states induced by the interaction of the first molecular monolayers on ferromagnetic substrates $\underline{\mathbf{1 9}} \mathbf{2 4}$ are expected to govern the spin polarization at the molecule-metal interface, leading to changes in the sign and magnitude of the magnetoresistance in spin-valve devices. The formation of spin-polarized hybrid states has been determined by spin-polarized spectroscopy methods $\underline{\mathbf{2 1}}, \underline{\mathbf{2 3}}$ and principleproven in nanosized molecular junctions, $\underline{\mathbf{1 9}}$ but not yet verified and implemented in large-area functional device architectures.

In this work, we demonstrate the control of the magnetoresistance sign in a fully functional molecular spin-valve system based on NaDyClq, a novel mononuclear quinoline molecule, combined with $\mathrm{Co}$ and $\mathrm{NiFe}$ as spin injector and detector electrodes. Contrary to other $\mathrm{Co} / \mathrm{AlO}_{x} / \mathrm{molecule}_{\mathrm{NiFe}}$ spin valve systems, $\underline{\mathbf{2 5}}-\underline{\mathbf{2 7}}$ the $\mathrm{Co} / \mathrm{AlO}_{\star} / \mathrm{NaDyClq} / \mathrm{NiFe}$ devices exhibit negative magnetoresistance, evidence of a negative spin polarization at the $\mathrm{NaDyClq} / \mathrm{NiFe}$ interface. Inverting the spin valve stack order $-\mathrm{NiFe} / \mathrm{AlO}_{x} / \mathrm{NaDyClq} / \mathrm{Co}$ - leads to positive magnetoresistance, which points out to a positive spin polarization at the $\mathrm{NaDyClq} / \mathrm{Co}$ interface. This change in spin polarization at the molecular/ferromagnetic interface is linked to the formation of specific hybrid electronic states, as confirmed by X-ray absorption spectroscopy. These results highlight the importance of the selection of specific molecule-metal interfaces to deterministically engineer spin-dependent macroscopic parameters (such as magnetoresistance) in spintronic devices.

\section{Results and Discussion}

\subsection{Characterization of the Molecular Layers}

\subsubsection{Molecular Precursor}

The structure of the $\mathrm{Na}\left[\mathrm{Dy}\left(5,7 \mathrm{Cl}_{2} \mathrm{q}\right)_{4}\right]$ molecule (where $5,7 \mathrm{Cl}_{2} \mathrm{q}=5,7$-dichloro-8hydroxiquinolate), in short $\mathrm{NaDyClq}$, is depicted in Figure $1 \mathrm{a}$. It features a central Dy atom surrounded by four $\mathrm{Cl}$-substituted quinolinolate ligands and a sodium countercation. While its molecular structure and energy band gap (optical gap of $2.7 \mathrm{eV}$, see Figure S1 in the Supporting Information) closely resembles $\mathrm{Alq}_{3}$-the most studied compound in the molecular spintronics community-, its magnetic properties are rather similar to the trinuclear family $\mathrm{Ln}_{3} \mathrm{q}_{9}, \underline{\mathbf{2 7}}$ in particular its single-molecule magnet behavior at low temperatures. It is found that the $\mathrm{NaDyClq}$ complex retains its molecular structure and magnetic properties upon sublimation, $\underline{\mathbf{2 8}}$ being thermally stable up to $380{ }^{\circ} \mathrm{C}$ according to thermogravimetry analysis (Figure $\underline{1} b$ ), which enabled the deposition of molecular layers on various substrates. The synthesis, sublimation, structural, and magnetic 
properties of this new mononuclear molecular family are presented in a separate detailed study. $\underline{\mathbf{2 8}}$

(a)

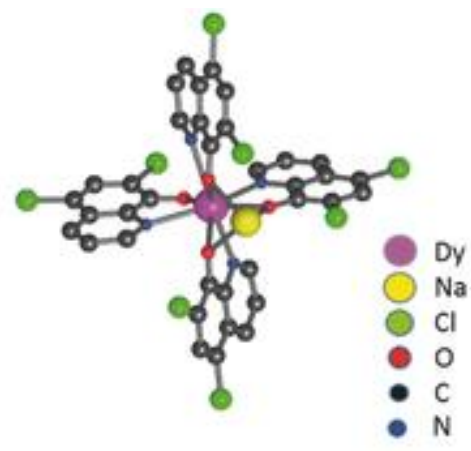

(c)

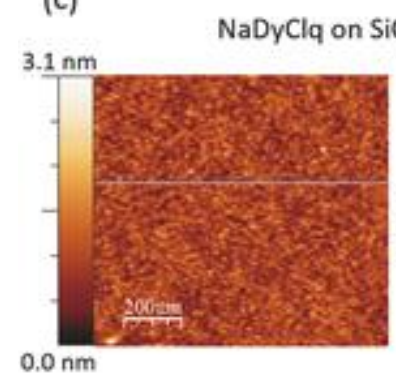

(b)

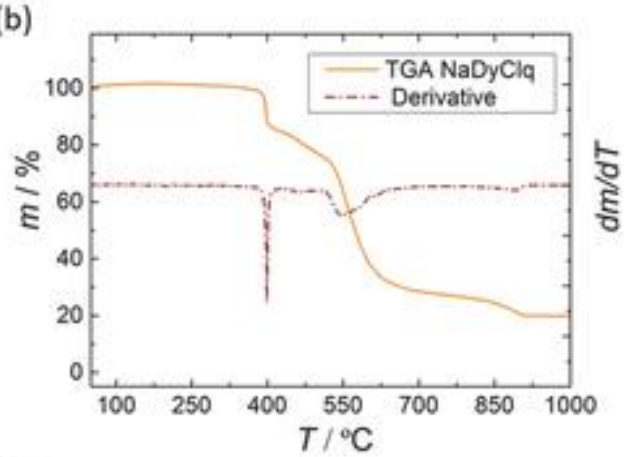

(d)

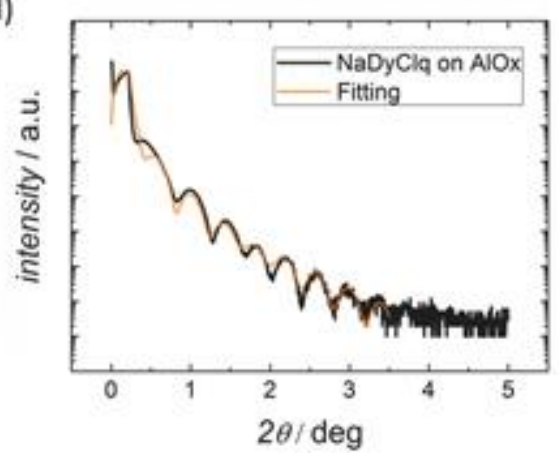

\section{Figure 1}

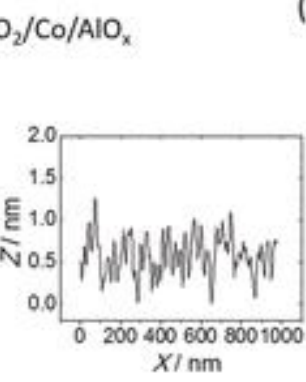

a) Molecular structure of the $\mathrm{Na}\left[\mathrm{Dy}\left(5,7 \mathrm{Cl}_{2} \mathrm{q}\right)_{4}\right]$ compound. b) Thermogravimetric analysis of the molecule, showing its thermal stability up to $380{ }^{\circ} \mathrm{C}$. The mass-loss derivative $\mathrm{d} m / \mathrm{d} T$ is shown on the right axis to visualize the phase change. c) AFM image of an $8 \mathrm{~nm}$ thick $\mathrm{NaDyClq}$ layer grown on $\mathrm{AlO}_{x}$ seed layer, including a topography cross section. d) $\mathrm{X}$-ray reflectivity scan of the $\mathrm{NaDyClq} / \mathrm{AlO}_{x}$ film, indicating high structural quality of the molecular layer on a long range scale.

\subsubsection{Optimization of NaDyClq Layer Properties}

For the fabrication of vertical spin-valve structures, the growth morphology of $\mathrm{NaDyClq}$ molecules on ferromagnetic layers is of crucial importance. It is found that the deposition of $\mathrm{AlO}_{x}$ thin layers $(1 \mathrm{~nm})$ on top of ferromagnetic metals greatly improves the surface quality for molecular growth, as evidenced by the comparison of $\mathrm{SiO}_{2} /$ ferromagnetic (FM) and $\mathrm{SiO}_{2} / \mathrm{FM} / \mathrm{AlO}_{x}$ surface topographies (Figure $\mathrm{S} 2$, Supporting Information). The oxide layer changes the surface energy of the bottom electrode and promotes a smoother growth of the molecular layer. The atomic force microscopy (AFM) image of an $8 \mathrm{~nm}$ thick $\mathrm{NaDyClq}$ layer grown on $\mathrm{SiO}_{2} / \mathrm{Co} / \mathrm{AlO}_{x}$ surface is shown in Figure $1 \mathrm{c}$. A remarkably smooth molecular surface topography can be observed, yielding root-meansquare roughness (RMS) values as low as $0.25 \pm 0.02 \mathrm{~nm}$, and peak-to-peak roughness as low as $1 \mathrm{~nm}$, which corresponds roughly to a molecular monolayer. Moreover, the structural and morphological quality of the molecular layer is preserved in a long-range scale, as evidenced from X-ray reflectivity (XRR) measurements. Figure $\underline{1} d$ shows the 
low-angle XRR scan a NaDyClq/AlO large-area thin film $(10 \mathrm{~mm} \times 10 \mathrm{~mm})$, featuring clear interference fringes which are indicative of clear and sharp top and bottom interfaces of the molecular layer. More quantitatively, the data has been fitted with a two-layer model $\left(\mathrm{AlO}_{x}+\mathrm{NaDyClq}\right.$, orange curve in Figure $\left.\underline{1} \mathrm{~d}\right)$ to extract layer properties such as thickness $(t)$, density $(\rho)$, and surface roughness, yielding following results for the NaDyClq layer: $t=8.7 \pm 0.5 \mathrm{~nm}, \rho=2.5 \pm 0.2 \mathrm{~g} \mathrm{~cm}^{-3}$, and a RMS roughness of $0.33 \pm$ $0.05 \mathrm{~nm}$. The extracted density values are a factor of two greater than the $\operatorname{Ln}_{3} \mathrm{q}_{9}$ trinuclear compounds, 27 indicating a rather closely packed molecular assembly. As for the surface roughness, the extracted RMS value $(0.33 \pm 0.05 \mathrm{~nm})$ of the NaDyClq layer is slightly higher than the results obtained by AFM, probably due to the large sampling area, but still good enough to grow continuous layers on the molecular surface. These results indicate that $\mathrm{AlO}_{x}$ is a convenient seed layer for the homogeneous and high-quality growth of the $\mathrm{NaDyClq}$ and their integration in vertical multilayer structures.

\subsection{Molecular Spin Valve Devices}

\subsubsection{Device Integration Scheme}

Molecular spin valves consist of a molecular layer inserted between two FM leads, which serve as spin injector and detector, respectively. In our case, the device consists on the following vertical stack:

FM1 $(15 \mathrm{~nm}) / \mathrm{AlO}_{x}(1 \mathrm{~nm}) / \mathrm{NaDyClq}(x \mathrm{~nm}) / \mathrm{FM} 2(15 \mathrm{~nm})$,

where FM1 and FM2 can be either NiFe or Co, respectively, and $x$ is the nominal thickness of the molecular layer and ranges from 8 to $12 \mathrm{~nm}$. A critical issue for the fabrication of molecular spin valves in a vertical geometry is that the evaporation of metals on top of molecules can lead to penetration of the metal atoms toward the bottom electrode (pinholes), especially in case of high roughness and/or sparse packing of the molecular layers. Some approaches have been employed to circumvent this issue, such as the insertion of $\mathrm{Al}_{2} \mathrm{O}_{3}$ interlayers at the top metal/molecule interface 29 or the development of gentle, off-angle metal deposition methods. $\underline{30}, \underline{31}$ In our case, the key factor was to grow an $\mathrm{AlO}_{x}$ seed layer on top of the bottom Cobalt (Co) or Permalloy $\left(\mathrm{Ni}_{80} \mathrm{Fe}_{20}, \mathrm{NiFe}\right.$ in short) electrodes, in order to achieve optimal structural properties of the molecular layer (surface roughness, packing density), as discussed in the previous section. The result of the multilayer growth optimization was reflected in a clear magnetoresistance signal. In this regard, the existence of single, sharp switching events for each ferromagnetic electrode implies a magnetization reversal process typical from homogeneous, smooth magnetic layers, underlining the good quality of the NiFe layer grown on top of the molecules. Moreover, it suggests that the ferromagnetic electrodes are spatially well separated and thus magnetically decoupled. Both factors are in line with the presence of a homogeneous, sharp $\mathrm{NaDyClq} / \mathrm{NiFe}$ interface, exempted from metal interdiffusion or embedded magnetic clusters. The charge and magneto-transport results will be presented and discussed in the next section.

\subsubsection{Electrical Transport}


A sketch of the vertical structure of the spin valves and the four-point geometry used for the electrical transport measurements is shown in Figure 2 a. In each fabrication round, a reference $\mathrm{Co} / \mathrm{AlO}_{x} / \mathrm{NiFe}$ junction has been included, in order to address the contribution of the $\mathrm{AlO}_{x}$ layer to the overall junction resistance. Unlike typical $\mathrm{Al}_{2} \mathrm{O}_{3}$ tunnel barriers, our reference junctions exhibit resistances of a few $\Omega$, their electrical properties resemble the ones of a bad metal and they do not contribute to the magnetoresistance (MR) of the junction, as shown in Figure $\underline{2} b$ (blue curve). Furthermore, Figure $\underline{2} b$ shows the temperature dependence of the resistance of a typical NaDyClq spin valve $(x=8.0 \pm 0.5$

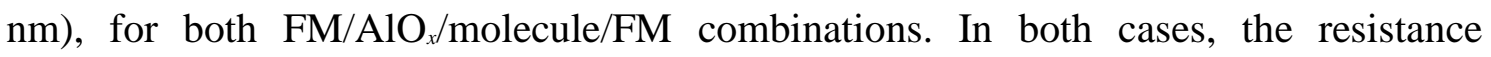
increases weakly with decreasing temperature (less than a factor of 3 ), which is mainly due to the Fermi-level broadening of the ferromagnetic leads. Figure $\underline{2} \mathrm{c}, \mathrm{d}$ displays the current-voltage $(I-V)$ characteristics at different temperatures for the two different FM electrode combinations. The $I-V$ curves are antisymmetric with respect to the origin and exhibit weak temperature dependence, features which are consistent with a tunneling transport mechanism. 25, $\underline{\mathbf{3 2}}$ However, it is known that the electrical transport in molecular systems undergo a transition from tunneling to hopping as a function of molecular length. $\underline{\mathbf{3 3}}, \underline{\mathbf{3 4}}$ In our molecular junctions, we rule out the occurrence of thermally activated hopping transport, as the $R(T)$ curves do not present an exponential temperature dependence in the studied thickness regime $(8-12 \mathrm{~nm})$.

(a)

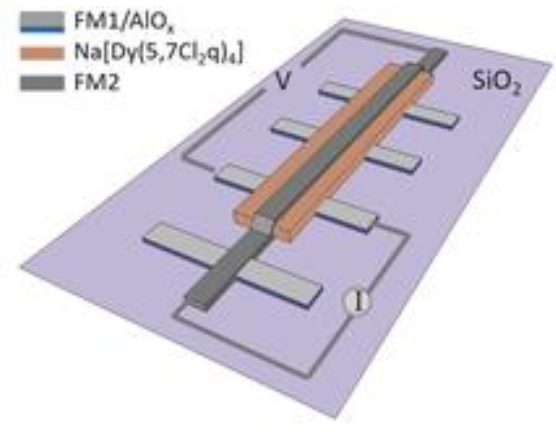

(c)

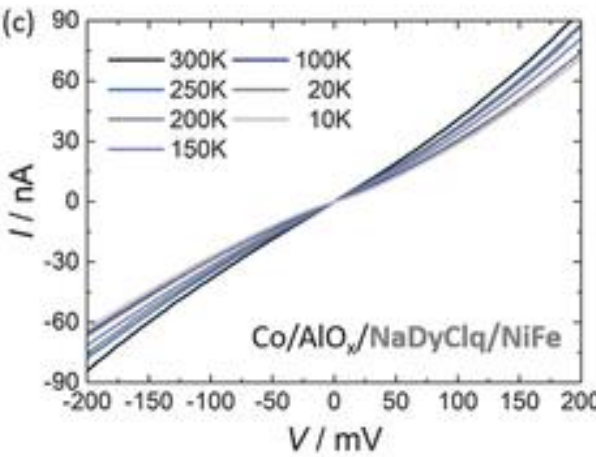

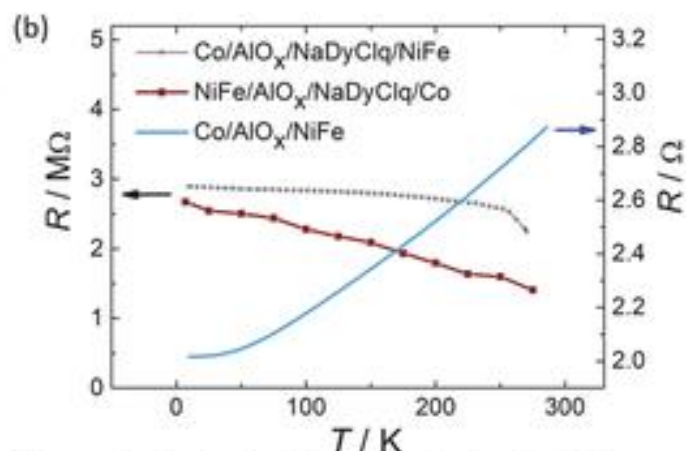

(d)

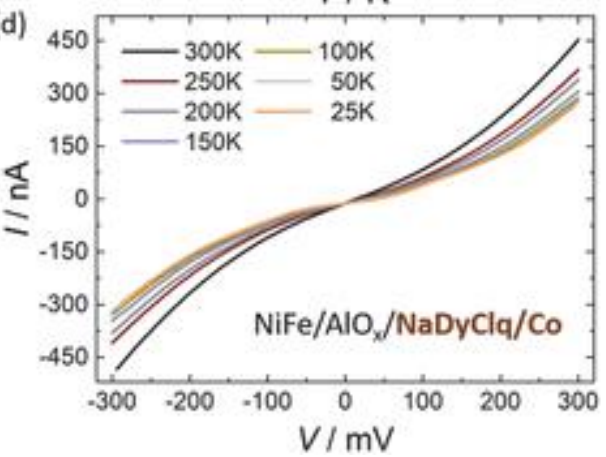

\section{Figure 2}

a) Sketch of the spin-valve device and 4-point measurement geometry. A reference junction without molecules is included in each chip. b) Temperature dependence of the resistance for a typical $\mathrm{NaDyClq}$ spin valve $(x=8.0 \pm 0.5 \mathrm{~nm})$ with both FM combinations (blue and brown) and for a reference junction without molecules (gray). c,d) Current- 
voltage traces of the molecular spin valve samples shown in (b), resembling characteristics of tunneling transport.

\subsubsection{Magnetotransport}

In order to probe the spin-dependent transport of the molecular spin valves, the magnetic field dependence of the electrical resistance has been measured between 300 and $5 \mathrm{~K}$. In a typical spin-valve, given that the current passing through the device is able to preserve its spin-polarization, two different resistance states are observed depending on the relative magnetic alignment of the injector and detector electrode. As we are in the regime of tunneling transport, the tunneling magnetoresistance is defined by tunneling magnetoresistance $(\mathrm{TMR})=\left(R_{\mathrm{AP}}-R_{\mathrm{P}}\right) / R_{\mathrm{P}}$, where $R_{\mathrm{P}}$ and $R_{\mathrm{AP}}$ are the measured resistances by a parallel and antiparallel alignment of the magnetic electrodes, respectively. Figure $\underline{3}$ a shows the magnetoresistance of an $8 \mathrm{~nm}$ thick $\mathrm{Co} / \mathrm{AlO}_{x} / \mathrm{NaDyClq} / \mathrm{NiFe}$ spin valve. Well-defined resistance states are observed in both trace and retrace field sweeps, and the transitions match nicely with the expected coercive fields of the Co and NiFe electrodes (Figure S6, Supporting Information). Importantly, the sign of the magnetoresistance is negative $\left(R_{\mathrm{AP}}<R_{\mathrm{P}}\right)$, unlike other vertical molecular spin valve structures using the same $\mathrm{Co} / \mathrm{AlO}_{x}$ and $\mathrm{NiFe}$ electrodes and $\mathrm{C}_{60}, \underline{\mathbf{2 5}}, \underline{\mathbf{3 2}}$ bathocuproine (BCP), $\underline{\mathbf{2 6}}$ and $\mathrm{Ln}_{3} \mathrm{q}_{9} \underline{\mathbf{2 7}}$ molecules as spin transport channels, which display a positive TMR. A change of sign in the magnetoresistance response in the tunneling regime might occur due to a reversal of the majority spin population at the Fermi-energy at one of the interfaces, an effect that has been first observed in inorganic magnetic tunnel junctions due to metal-oxide interface bonding $\underline{35}$ and later in molecular nanojunctions due to hybridization of the metal and molecular orbitals. $\underline{\mathbf{1 9}}$

(a) $\mathrm{Co} / \mathrm{AlO}, / \mathrm{NaDyClq} / \mathrm{NiFe}$

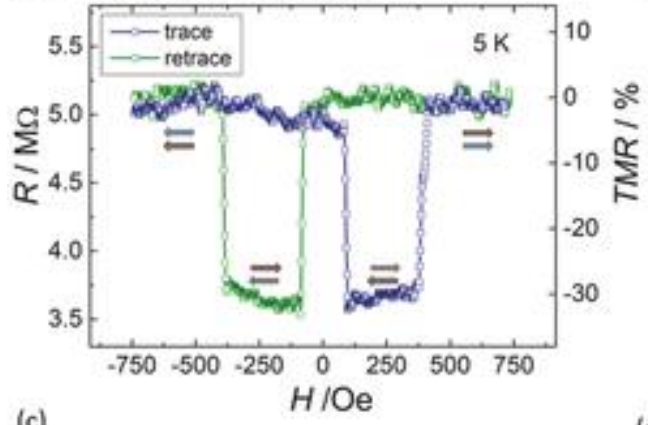

(c)

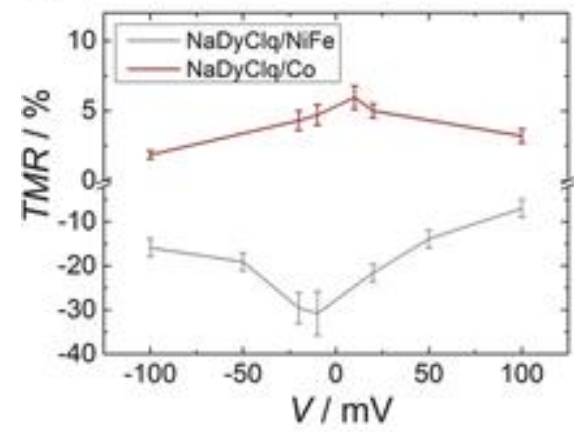

(b)

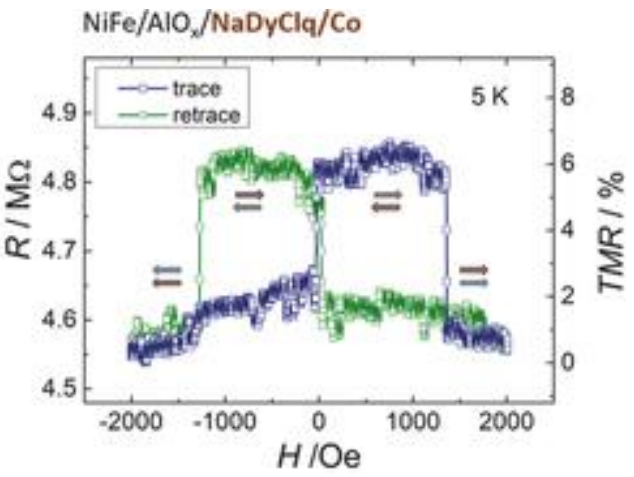

(d)

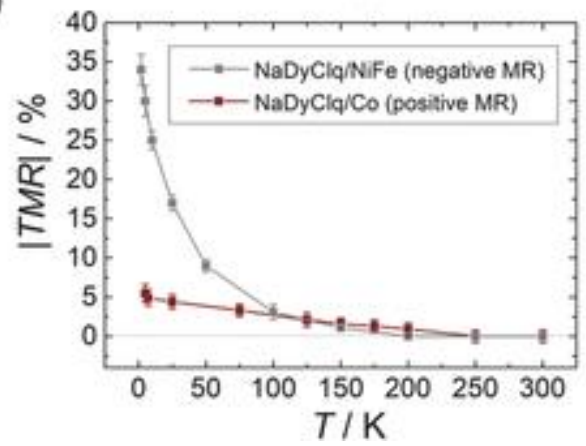




\section{Figure 3}

Magnetotransport measurements in NaDyClq spin valves and the role of the metalmolecule interface. a) Negative TMR measured at $5 \mathrm{~K}$ and $-10 \mathrm{mV}$ in a $\mathrm{Co} / \mathrm{AlO} / \mathrm{NaDyClq} / \mathrm{NiFe}$ spin valve. b) Positive TMR measured under the same conditions in a NiFe/AlO $/ \mathrm{NaDyClq} / \mathrm{Co}$ spin valve, highlighting the role of the top metalmolecule interface. The brown and gray arrows indicate the magnetization direction of the $\mathrm{Co}$ and $\mathrm{NiFe}$ electrodes respectively. c) Magnetoresistance as a function of injection bias for the cases described in (a) and (b), characteristic of tunneling transport. d) Absolute value of the tunneling magnetoresistance as a function of temperature, exhibiting a different scaling for devices showing positive or negative TMR. In both cases, the TMR effect goes to zero well below room temperature (around $200 \mathrm{~K}$ ).

To further understand the role of the hybrid interfaces on the sign inversion of the TMR, we have investigated the metal-molecule interaction at a second interface, $\mathrm{NaDyClq} / \mathrm{Co}$, by swapping the order of the ferromagnetic electrodes in the spin-valve, as $\mathrm{NiFe} / \mathrm{AlO}_{x} / \mathrm{NaDyClq} / \mathrm{Co}$. In this way, the bottom $\mathrm{NiFe}$ electrode is physically decoupled from the molecule, leaving the $\mathrm{NaDyClq} / \mathrm{Co}$ as the active interface. As shown in Figure $\underline{3} b$, we measure a positive magnetoresistance, which indicates that the top $\mathrm{NaDyClq} / \mathrm{Co}$ interface has the same spin polarization as the bottom NiFe electrode. This demonstrates that the sign of the MR is strongly dependent on the metal-molecular combination and the resulting hybrid spin-polarized density of states relevant for tunneling magnetoresistance. Furthermore, as the spin-polarized density of states (DOS) is extremely sensitive to the degree of admixture of the metal and molecular states at the corresponding interfaces, it is clear that the magnitude of the positive TMR $(5.5 \pm 0.8 \%$, Figure $\underline{3} \mathrm{~b}-\mathrm{NaDyClq} / \mathrm{Co})$ is notably different than the negative one $(-31 \pm 2 \%$, Figure $\underline{3} \mathrm{a}$ - NaDyClq/NiFe). The injection bias dependence of the magnetoresistance is shown in Figure $\underline{3} \mathrm{c}$ for both active interfaces, evidencing a slightly asymmetric behavior ascribed to the asymmetric density of spin-polarized states encountered by the tunneling electrons when injected from the metal/AlO $x$ or the metal electrode into the molecules. 32 The shape of the TMR $(V)$ curve is preserved as the temperature is increased (Figure S3, Supporting Information), demonstrating that the spin-polarized injection process is entirely determined through the applied voltage at each of the electrodes rather than by thermally assisted effects. 32 It is also worth noting that the sign of the TMR (either negative or positive) is maintained for both samples in the studied bias regime $(<100 \mathrm{mV}$, Figure $\underline{3} \mathrm{c}$; Figure S3, Supporting Information). As for the temperature dependence of the TMR, as shown in Figure $\underline{3} \mathrm{~d}$, it is found to differ drastically upon the choice of the metal-molecule interface. For the negative TMR sample, which has a large TMR magnitude at low temperature, the effect drops rather rapidly with increasing temperature, while for the positive TMR sample the decrease is rather smooth and nearly linear. As both devices had a very similar temperature dependence of the charge transport (Figure $\underline{2} b$ ), we attribute this difference to be a direct consequence of the resulting temperature dependent spin-polarization at the hybrid interface. Moreover, the TMR disappears well below 
room-temperature in both cases, suggesting that the spin-dependent density of states at any of the active interfaces cannot be described by the one of the ferromagnet. These findings can be explained in terms of a lower "effective" Curie temperature at the metalmolecule interface.

\subsubsection{Control Experiments for MR Inversion Phenomena}

In inorganic tunnel junctions, a sign change of the magnetoresistance has been observed upon insertion of a metal interlayer (such as $\mathrm{Cu}, \mathrm{Au}$, or $\mathrm{Al}$ ) as a consequence of the resonant tunneling of quantum well states. $\underline{\mathbf{3 6}}, \underline{\mathbf{3 7}}$ This is a priori not expected in our devices since he $\mathrm{AlO}_{x}$ seed layer does not have an ordered crystalline structure and is thus not a suitable system for the formation of resonant tunneling states. Moreover, an inversion of the MR has been reported in organic spin valves based on $\mathrm{Alq}_{3}$ by the insertion of a metallic $\mathrm{Al}$ layer $(0-5 \mathrm{~nm})$ at the $\mathrm{Alq}_{3} / \mathrm{Co}$ interface. $\mathbf{3 8}$ In order to investigate if an excess $\mathrm{Al}$ layer due to insufficient oxidation at our bottom $\mathrm{Co} / \mathrm{AlO}_{x}$ interface inverts the sign of the TMR, a series of $\mathrm{Co} / \mathrm{Al} / \mathrm{Al}_{2} \mathrm{O}_{3} / \mathrm{NiFe}$ magnetic tunnel junctions (MTJs) have been prepared (see Figure 4; Figure S4, Supporting Information). We choose the $\mathrm{Co} / \mathrm{Al}_{2} \mathrm{O}_{3} / \mathrm{NiFe}$ magnetic tunnel junction as a reference, as it unambiguously presents a positive MR and is thus a good reference system for this study. The tunneling behavior in the junctions is assessed by $I-V$ characteristics, as shown in Figure $\underline{4}$ a. Most importantly, a positive magnetoresistance is always observed up to an Al thickness of 1 $\mathrm{nm}$ (Figure $\underline{4} \mathrm{~b}$ ), which corresponds to the extreme case of having our full $\mathrm{AlO}_{x}$ seed layer (1 nm) unoxidized. In addition, we did neither observed any TMR sign change by performing the same experiment with the $\mathrm{AlO}_{x}$ barrier used for the molecular spin valves instead of pure Al. Additionally, it has been cross-checked that the TMR preserves the positive sign at low temperatures $(5 \mathrm{~K})$, as depicted in Figure S4 in the Supporting Information. These control experiments rule out any excess $\mathrm{Al}$ layer as well as the $\mathrm{AlO}_{x}$ "leaky" barrier itself as the responsible for the TMR sign inversion in the molecular spin valves.

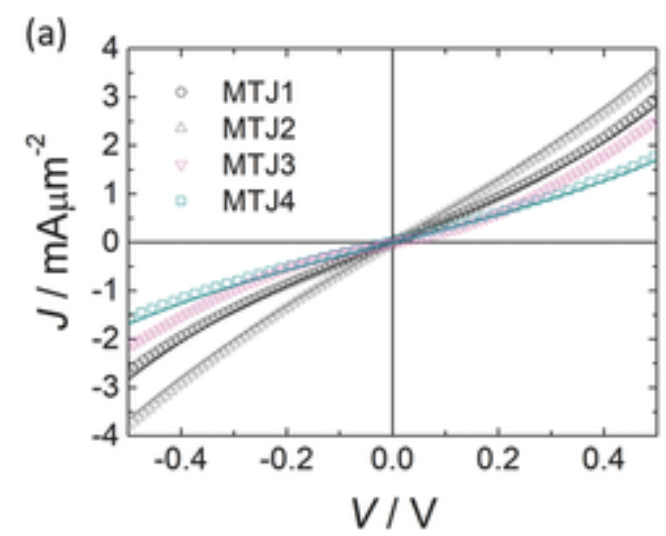

(b)

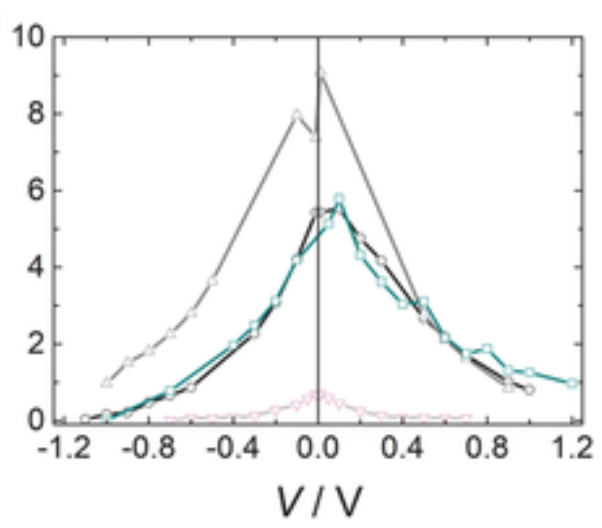

Figure 4 
a) $I-V$ curves of the magnetic tunnel junctions, showing tunneling transport characteristics of the devices: $\mathrm{MTJ} 1\left(\mathrm{Co} / \mathrm{Al}_{2} \mathrm{O}_{3}(1.5 \mathrm{~nm}) / \mathrm{NiFe}\right), \mathrm{MTJ} 2(\mathrm{Co} / \mathrm{Al}(0.7$ $\left.\mathrm{nm}) / \mathrm{Al}_{2} \mathrm{O}_{3} 1.3 \mathrm{~nm} / \mathrm{NiFe}\right), \mathrm{MTJ} 3\left(\mathrm{Co} / \mathrm{AlO}_{x}(1 \mathrm{~nm}) / \mathrm{Al}_{2} \mathrm{O}_{3} 1.5 \mathrm{~nm} / \mathrm{NiFe}\right)$, and MTJ4 (Co/Al $(1 \mathrm{~nm}) / \mathrm{Al}_{2} \mathrm{O}_{3} 2 \mathrm{~nm} / \mathrm{NiFe}$ ). b) Dependence of the tunneling magnetoresistance (TMR) signal with applied voltage at room temperature, showing no sign reversal of the TMR up to $1 \mathrm{~V}$ for all the junctions.

Another scenario leading to a TMR sign inversion relies on the formation of new interfacial phases due to a strong chemical reaction at the metal-molecule interface. It has been previously shown that such phases might exhibit a drastically different magnetic behavior and thus alters the magnetotransport properties in hybrid tunnel junctions. $\underline{\mathbf{3 9}}$ Specifically, in Ref. $\underline{\mathbf{3 9}}$ an inversion of the magnetoresistance was observed upon formation of an antiferromagnetic $\mathrm{CoF}_{2}$ layer at the $\mathrm{Co} / \mathrm{LiF}$ interface used as spin injector in a hybrid spin valve. In our case, a hypothetic strong chemical reaction would rather create $\mathrm{Cl}$-based compounds, as the $\mathrm{NaDyClq}$ molecule features chlorine-rich ligands (see Figure $\underline{1}$ a, in green). Possible compounds are $\mathrm{NiCl}_{2}, \mathrm{FeCl}_{2}$ and $\mathrm{FeCl}_{3}$, which are antiferromagnetic with ordering temperatures of 50,24 , and $10 \mathrm{~K}$, respectively. $\mathbf{4 0}$ 42 As the negative TMR effect is present above $150 \mathrm{~K}$ in our NaDyClq spin valves, we rule out the formation of such interfacial antiferromagnetic compounds as the origin of the negative magnetoresistance effect. A third scenario for a negative MR is the existence of a static antiferromagnetic coupling between the metal and the molecular moments. For instance, Gruber et.al.11 report an antiferromagnetic coupling up to $100 \mathrm{~K}$ in a $\mathrm{MnPc} / \mathrm{Co}$ system. If this were the case in our metal-molecule system, we would expect a transition from a negative to a positive magnetoresistance as we go over the Nèel temperature of the interfacial system. But the observation of a pure negative TMR up to $150 \mathrm{~K}$ rules out this possibility. In turn, all the experimental observations described above point toward the scenario of a TMR sign change due to the formation of hybrid states at the $\mathrm{NaDyClq} / \mathrm{NiFe}$ interface.

\subsection{Investigation of the NaDyClq/Metal Interfaces}

\subsubsection{X-Ray Absorption Spectroscopy of In Situ Grown Samples}

In order to investigate the microscopic details of this hybridization process, we have performed in situ X-ray absorption spectroscopy (XAS) measurements at the $\mathrm{NaDyClq} / \mathrm{NiFe}$ and $\mathrm{NaDyClq} / \mathrm{Co}$ interfaces. X-ray absorption spectra were taken at all the edges of the elements composing the NaDyClq molecule as well as at the ferromagnetic metal $\mathrm{L}_{2,3}$ edges both in grazing and normal incidence with linearly polarized light, immediately before and after the $\mathrm{NiFe}(\mathrm{Co})$ ultra-high vacuum (UHV) deposition step. While the $\mathrm{Na}$ and $\mathrm{Cl}$ spectra exhibit a low signal-to-noise ratio due to their low absorption cross-sections, all the other edges ( $C, N, O, D y)$ could be acquired 
with good quality. In the as-grown $\mathrm{NaDyClq}$ molecule, the spectra taken at normal $\left(90^{\circ}\right.$, in-plane polarization) and grazing incidence (20 $0^{\circ}$, almost out-of-plane polarization) with linearly polarized X-rays, exhibit identical features with slightly varying intensity ratios (Figure S7, Supporting Information). These results indicate that the electronic orbitals do not have a preferential spatial orientation, in agreement with the spherically symmetric 3D character of the quinoline compound. For the following analysis, we just consider the spectra taken in grazing incidence, as they imply a larger probing area on the specimen surface, leading to a simultaneous gain in intensity and interface sensitivity.

Figure $\underline{5}$ compares the normalized $\mathrm{C}$ K-edge and N K-edge XAS spectra of the NaDyClq layer before and after metal deposition, confirming that each metal surface ( $\mathrm{NiFe}$ and $\mathrm{Co}$ ) leads to a different, specific interaction with the molecular orbitals. While the $\mathrm{NiFe}$ adsorption notably modifies the carbon K-edge spectrum (Figure $\underline{\mathbf{5}} \mathrm{a}, \mathrm{b}$ ), Co atoms rather interact with the $\mathrm{N}$-sites of the molecule, as seen in the changes of the $\mathrm{N}$ K-edge absorption spectrum (Figure $\underline{\mathbf{5}} \mathrm{c}, \mathrm{d}$ ). In the carbon K-edge of the pristine molecular layer, the electronic transitions belonging to both $\pi$ and $\sigma$ carbon orbitals can be clearly identified (Figure $\underline{5} b)$. In the case of the $\pi$ states, there are three prominent peaks $\left(A_{1}-A_{3}\right)$, which have been previously observed in $\mathrm{Alq}_{3}$ molecules and ascribed to the lowest unoccupied molecular orbital (LUMO) and LUMO+1 transitions localized at the carbon rings of the quinolinolate ligands. $\mathbf{4 3}$ Comparing the spectrum before and after deposition of NiFe atoms, there are two remarkable differences. First, there are two sharp, highintensity peaks (highlighted with a star), that are ascribed to high-order replica from the Ni $\mathrm{L}_{2,3}$, absorption edge, and therefore are not related to molecule-metal interaction. Second, and most importantly, there is a clear increase in spectral weight in the pre-edge region, especially at $280.6 \pm 0.2$ and $282.0 \pm 0.3 \mathrm{eV}$ (Figure $\underline{5}$ b). While the $282.0 \mathrm{eV}$ feature is present in the pristine $\mathrm{NaDyClq}$ layer with a much lower intensity, the 280.6 $\mathrm{eV}$ peak is a unique feature of the $\mathrm{NaDyClq} / \mathrm{NiFe}$ interface. This change in the spectrum indicates electronic transitions of the core electrons to newly formed electronic states and can be understood in terms of a specific hybridization of $\mathrm{NiFe}$ and carbon orbitals, since the spectra of all other species of the NaDyClq molecule remain unperturbed (Figure S8, Supporting Information). 

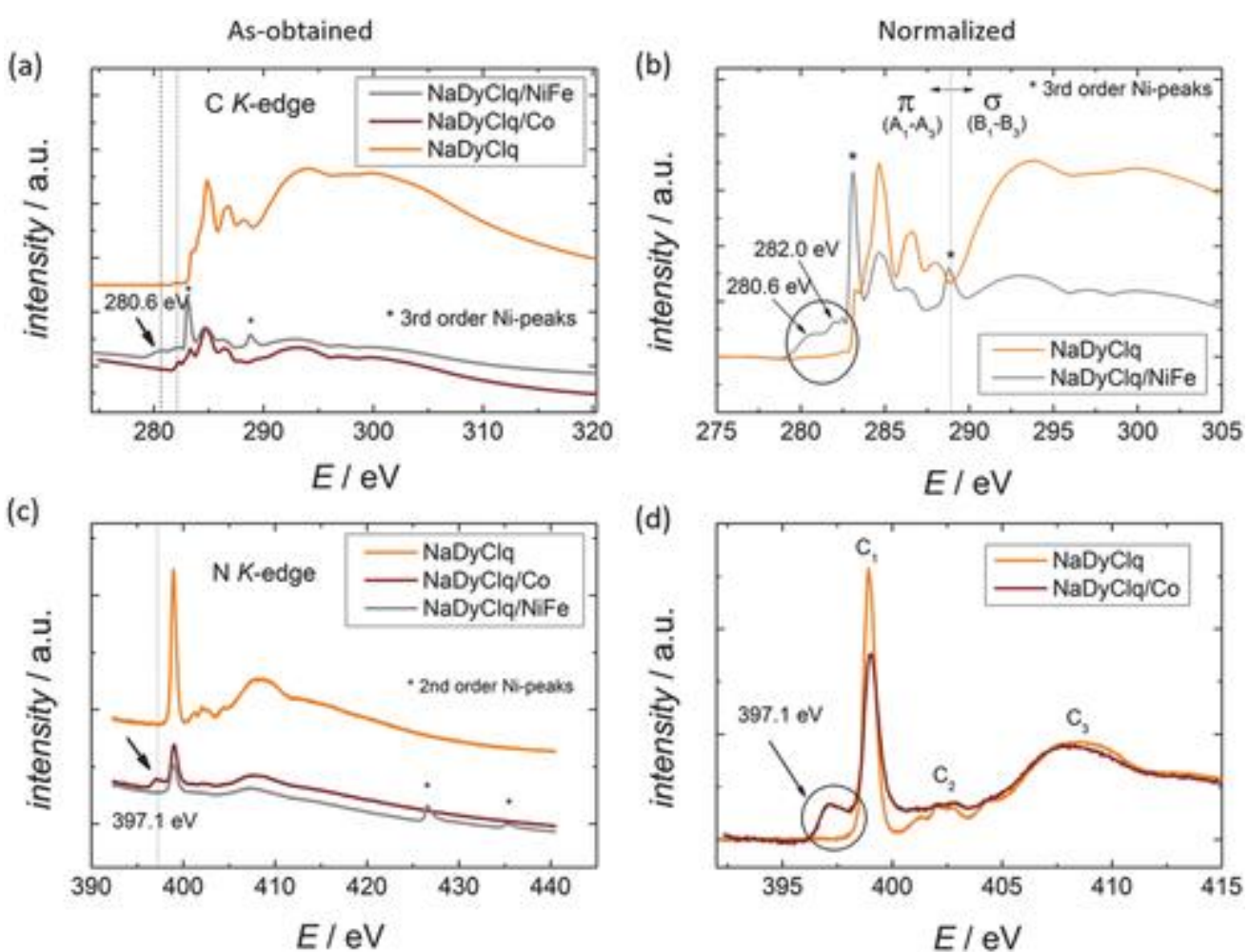

Figure 5

$\mathrm{X}$-ray absorption spectroscopy of the interacting elements at the two studied metalmolecule interfaces. a) As-obtained X-ray absorption spectra at the $\mathrm{C} \mathrm{K}$-edge, highlighting that the absorption features at 280.6 and $282.0 \mathrm{eV}$ arise specifically at the $\mathrm{NaDyClq} / \mathrm{NiFe}$ interface, while all other features are present in the pristine molecule, though with different relative intensities. Note that the additional sharp peaks marked with a star are higher order harmonic lines from the $\mathrm{Ni} \mathrm{L}_{2,3}$ edge. b) Normalized $\mathrm{C} \mathrm{K}$-edge spectrum highlighting the features in the pre-edge region upon $\mathrm{NiFe}$ deposition, in addition to the multiple transitions of the $\pi\left(\mathrm{A}_{1}-\mathrm{A}_{3}\right)$ and $\sigma\left(\mathrm{B}_{1}, \mathrm{~B}_{2}\right)$ orbitals. c) As-obtained $\mathrm{N}$ K-edge spectrum of the $\mathrm{NaDyClq} / \mathrm{Co}$ interface, presenting a clear feature related to $\mathrm{Co}-\mathrm{N}$ bonding states, which is not present at the $\mathrm{NaDyClq} / \mathrm{NiFe}$ interface. The normalized spectrum is shown in (d), along with the known $\mathrm{C}_{1}-\mathrm{C}_{3}$ transitions of the $\mathrm{N}$ orbitals.

The situation differs in the case of Co atoms deposited onto the NaDyClq layer. While the carbon K-edge spectrum displays the same features as the pristine $\mathrm{NaDyClq}$ molecule, the N K-edge displays a clear new peak in the pre-edge region at $397.1 \pm 0.1 \mathrm{eV}$ (Figure $\underline{5} \mathrm{c}, \mathrm{d})$, in addition to the well-known transitions of the $\pi\left(\mathrm{C}_{1}\right)$ and $\sigma\left(\mathrm{C}_{2}, \mathrm{C}_{3}\right)$ orbitals. A similar increase of the spectral weight on the pre-edge region of the $\mathrm{N} \mathrm{K}$-edge has been previously observed by the deposition of MnPc molecules on a Co substrate and ascribed to the formation of hybrid Co-N states. 44 Also in our case, the interaction of Co is found to be specific to the nitrogen atoms of the quinoline molecule, which underlines the unique character of interface bonding at each molecule-metal interface. Interestingly, these states present a clear linear dichroism, as shown in Figure $\underline{\mathbf{6}}$, feature which is not present in the purely $(\pi, \sigma)$ molecular states, pointing toward a geometrical orbital asymmetry originated from the $\mathrm{Co}-\mathrm{N}$ hybridization process. 

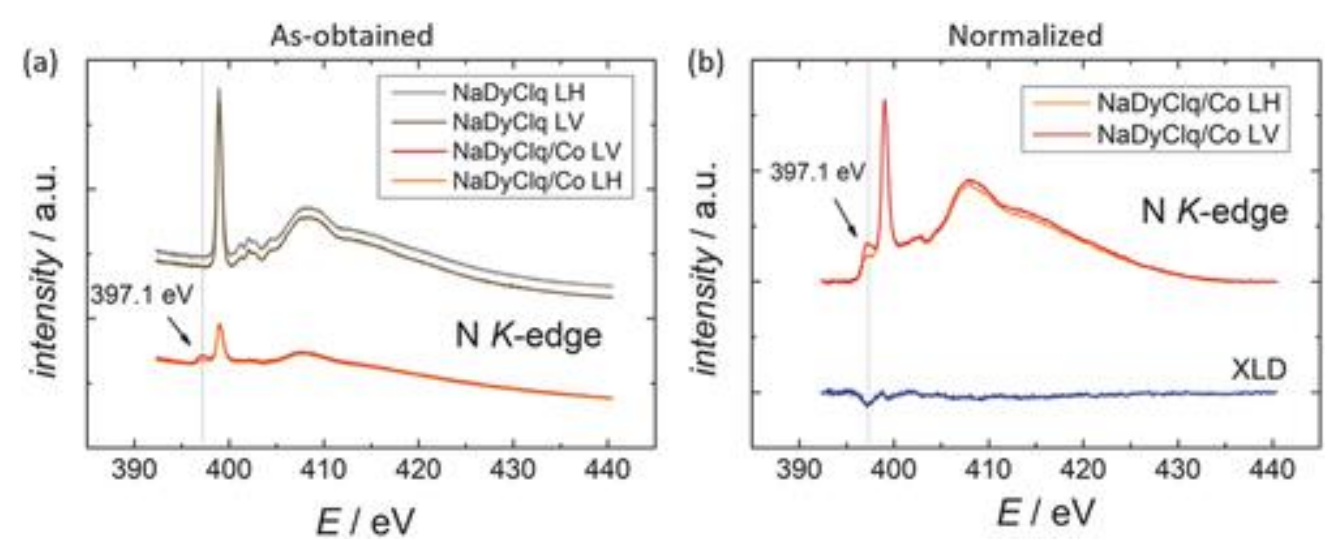

Figure 6

a) As-obtained X-ray absorption spectra at the $\mathrm{N} \mathrm{K}$-edge for linearly horizontal (LH) and linearly vertical (LV) polarized light, before and after the codeposition step. The emergence of new Co-N hybrid states exhibiting linear dichroism is highlighted in panel (b), after background subtraction.

\subsubsection{Correlation Between Spectroscopy and Magnetotransport}

The spectroscopic data presented above is consistent with a chemical interaction and the formation of specific hybrid electronic states at the $\mathrm{NaDyClq} /$ metal interface. As for the implications to the spin-valve devices, the fact that these hybrid states lie energetically below the molecular LUMO (that is in the bandgap, see Figure S9 in the Supporting Information), underlines their contribution to the density of states at the injector/detector interface, which redefines the spin-polarization relevant for tunneling magnetoresistance. Furthermore, the connection between hybrid interfacial states and spin transport can be also hinted on the temperature dependence of the TMR (Figure $\underline{3} \mathrm{~d}$ ) for the spin valves with both $\mathrm{NaDyClq} / \mathrm{FM}$ active interfaces. The signals disappear well below room temperature, indicating that the spin polarization of the hybrid interface states is different from the one of the bulk ferromagnet, even changing its sign. The existence of an "effective interface Curie temperature" seems plausible considering the hybridization of carbon or nitrogen orbitals with the s- and/or d-states of the ferromagnet as deduced by the XAS measurements. A complete microscopic description of the relation between the hybridization and the TMR should include full first-principles calculation together with transport calculations associated with it.

\section{Conclusions}

In summary, molecular spin-valves based on novel lanthanide-based quinolines of the

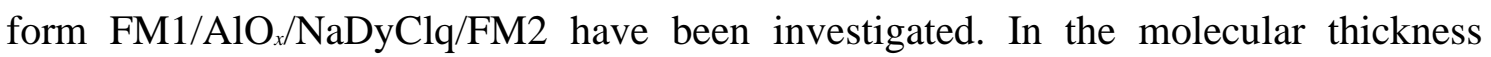
regime studied $(8-12 \mathrm{~nm})$, tunneling has been identified as the relevant transport mechanism. As for the magnetotransport properties, the $\mathrm{Co} / \mathrm{AlO}_{x} / \mathrm{NaDyClq} / \mathrm{NiFe}$ devices exhibit a negative magnetoresistance, whereas swapping the NiFe and Co electrodes leads to a positive TMR signal. This effect is found to be related to the formation of specific 
hybrid states at the $\mathrm{NaDyClq} / \mathrm{NiFe}$ (Co) interface, as confirmed by in situ $\mathrm{X}$-ray absorption spectroscopy. Element-specific X-ray absorption spectra reveal the existence of interface-specific hybridized $\mathrm{Co}-\mathrm{N}$ and $\mathrm{NiFe}-\mathrm{C}$ states, both of which are found to be energetically below the LUMO of the molecule and hence contributing to the electronic and spin-dependent transport of the spin valves. Our combined transport and spectroscopic study of the spin valve structures demonstrate how a specific interaction between a ferromagnetic metal and a molecule can change macroscopic device parameters such as the sign and magnitude of magnetoresistance. These findings underline the huge potential of molecules to tailor spin-dependent properties at metal interfaces and most importantly, transduce this microscopic interaction into device output signals, ultimately proving the spinterface $\underline{\mathbf{2 0}}$ concept in functional large-area spintronic devices.

\section{Experimental Section}

Molecule Synthesis: The molecular compound $\mathrm{Na}\left[\mathrm{Dy}\left(5,7 \mathrm{Cl}_{2} \mathrm{q}\right)_{4}\right]$ was synthesized as follows. All materials and reagents were purchased from Sigma-Aldrich. A solution (4 mmol) of the recrystallized ligand 5,7-dichloro-8-hydroxyquinoline in chloroform was dissolved of absolute ethanol $(80 \mathrm{~mL})$ at $50{ }^{\circ} \mathrm{C}$ under stirring. Then, a solution $(4 \mathrm{mmol})$ of $\mathrm{NaOH}$ in absolute ethanol was added at $50{ }^{\circ} \mathrm{C}$. Subsequently, another solution of $\mathrm{LnCl}_{3} .6 \mathrm{H}_{2} \mathrm{O}(0.6 \mathrm{mmol})$ in absolute ethanol at $50{ }^{\circ} \mathrm{C}$ was slowly added dropwise and kept under stirring at $50{ }^{\circ} \mathrm{C}$ during $1 \mathrm{~h}$. Later, the solution was stirred at room temperature. The yellow precipitate was filtered and washed with milli-pore water and a cold mixture $\mathrm{EtOH} / \mathrm{H} 2 \mathrm{O}[1: 1](10 \mathrm{~mL})$. Crystals were dried at $110 \mathrm{C}$ during one day.

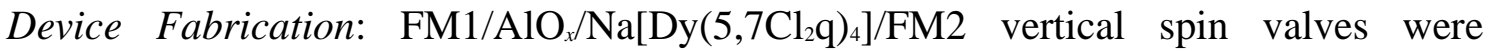
fabricated at UHV in an in situ dual chamber evaporator at a base pressure of $10^{-11} \mathrm{mbar}$, using shadow masks. Ferromagnetic electrodes were evaporated at a rate of $1 \AA^{-1}$ via electron beam evaporation. Aluminum was e-beam evaporated at $0.1-0.2 \AA^{-1}$ and oxidized in a controlled atmosphere at $0.1 \mathrm{mbar}\left(\mathrm{O}_{2}\right.$ pressure $)$ during $20 \mathrm{~min}$ to conform a partially oxidized (leaky) $\mathrm{AlO}_{x}$ barrier. The $\mathrm{Na}\left[\mathrm{Dy}\left(5,7 \mathrm{Cl}_{2} \mathrm{q}\right)_{4}\right]$ layers were sublimated thermally from conventional effusion cells, at evaporation temperatures ranging between 345 and $355^{\circ} \mathrm{C}$, keeping the substrate at a constant temperature of $24^{\circ} \mathrm{C}$. The growth rate and thus the molecular layers' thicknesses were determined by reference samples calibrated by X-ray reflectivity. The obtained active device areas were in the range $200 \times$ $100-200 \times 300 \mu \mathrm{m}^{2}$, and have been structured via in situ shadow masking.

Characterization Methods: The thermogravimetric and differential thermal analyzer used is a Mettler Toledo TGA/SDTA $851 \mathrm{e}$ that operates in the range $25-1100{ }^{\circ} \mathrm{C}$ with a 
sensibility of $0.1 \mu \mathrm{g}$. The structural and chemical integrity of the molecular layers has been verified via infrared spectroscopy, using a Bruker Hyperion 2000 microscope coupled to a Vertex 70 Fourier-transformed infrared spectrometer (FTIR) spectrometer equipped with a mercury cadmium telluride (MCT) detector, with a resolution of 4 $\mathrm{cm}^{-1}$ and the average of 1000 interferograms. Surface topographies were imaged using an atomic force microscopes (Agilent 5500), operating in tapping mode with a tip frequency of $342 \mathrm{kHz}$. Cantilevers were from silicon, with a spring constant of $40 \mathrm{Nm}^{-1}$. The image area amounts to $1 \mu \mathrm{m} \times 1 \mu \mathrm{m}$. Electrical and magnetotransport measurements were performed in vacuum in a Lakeshore Probe Station with variable temperature in the range 5-300 K and in-plane magnetic field up to 0.6 T, as well as with a state of the art Quantum Design Physical Properties Measurement System, with a temperature range from 2 to 300 K. For the current-voltage and MR curves a Keithley 4200 semiconductor parameter analyzer was used. $M$ versus $H$ curves was measured with a quantum design squid magnetometer (Quantum Design MPMS), with a sensitivity of $5 \times 10^{-8} \mathrm{emu}$. The X-ray absorption spectroscopy measurements were carried out at the BOREAS beamline in ALBA $\underline{45}$ Synchrotron, Barcelona, equipped with a preparation chamber (low $10^{-9}$ mbar range) where the $\mathrm{NaDyClq} /$ metal layers were prepared in situ. The molecules were grown with the effusion cells described above and the $\mathrm{NiFe}$ and $\mathrm{Co}$ metals via e-beam evaporation, under the same conditions as in the in-house lab for the device fabrication. The X-ray absorption measurements were taken in total electron yield mode both in normal $\left(90^{\circ}\right)$ and grazing $\left(20^{\circ}\right)$ incidence of the photon beam with linearly polarized $\mathrm{X}$ rays; the spectra were normalized to the incident photon flux measured by a freshly evaporated Au-grid inserted in the X-ray path before the sample.

\section{Acknowledgements}

A.B.-P. and S.G.M. contributed equally to this work. The authors thank Roger Llopis for technical support at CiC nanoGUNE, as well as Gunnar Ohrwall from the beamline I1011 in Max Lab, Lund, for support in the acquisition of preliminary X-ray absorption data. Financial support from the EU (COST Action in Molecular Spintronics 15128, Projects HINTS FP7-263104, Spintros ERC Starting Grant ERC-2009-257654, and SpinMol ERC Advanced Grant ERC-2009-AdG-20090325), the Spanish Ministerio de Economía y Competitividad (Unit of Excellence Maria de Maeztu MDM-2015-0538 and MDM-20160618 as well as projects with FEDER cofinancing MAT2011-22785 MAT2012-37638, FIS2013-45469-C4-3-R, MAT2014-56143-R, and MAT2015-65159-R), Generalitat Valenciana (PROMETEO programme). S.G.M. thanks the Spanish MINECO for an FPI predoctoral grant. The authors acknowledge beamtime access at ALBA BL29 via proposal 2015-021263. 